\title{
VERSION KÄHLÉRIENNE D’UNE CONJECTURE DE ROBERT J. ZIMMER
}

\author{
PAR SERGE CANTAT
}

RÉSUMÉ. - Nous démontrons une conjecture de Robert J. Zimmer dans le cas particulier des actions de réseaux par difféomorphismes holomorphes sur les variétés kählériennes compactes.

(C) 2004 Published by Elsevier SAS

ABSTRACT. - Let $M$ be a compact Kähler manifold. Let $G$ be a connected simple real Lie group. Let $\Gamma$ be a lattice in $G$. We prove the following: if the $\mathbf{R}$-rank of $G$ is strictly larger than the complex dimension of $M$ any morphism from $\Gamma$ to the group of holomorphic diffeomorphisms of $M$ has finite image. This is a particular case in a conjecture of Robert J. Zimmer

(C) 2004 Published by Elsevier SAS

\section{Introduction}

Les travaux de Gregori A. Margulis permettent de comprendre les représentations linéaires des réseaux des groupes de Lie simples de rang réel strictement supérieur à 1 (voir $[15,17])$. Dans [18], Robert J. Zimmer propose de généraliser les travaux de Margulis au cadre des représentations non linéaires de réseaux. L'une des conjectures-phares du programme, attribuée à Zimmer, est la suivante (voir [18,19]).

Conjecture de Zimmer. - Soit $G$ un groupe de Lie réel simple connexe et $\Gamma$ un réseau de $G$. S'il existe un morphisme d'image infinie de $\Gamma$ dans le groupe des difféomorphismes d'une variété compacte $M$, le rang réel de $G$ est inférieur ou égal à la dimension de $M$.

Dans cet article, nous résolvons la conjecture de Zimmer pour le cas particulier des actions holomorphes sur les variétés kählériennes compactes. Ce cas particulier de la conjecture de Zimmer et les problèmes qu'il soulève nous avaient été signalés par Étienne Ghys il y a quelques années. La stratégie globale de la preuve et une large part des arguments développés dans les pages suivantes lui sont dus. Nous recommandons d'ailleurs la lecture de [10] pour le cas des actions de réseaux sur le cercle et celle de [6] pour des considérations analogues à celles que nous développerons ici.

THÉORÈmE A. - Soit $M$ une variété complexe compacte kählérienne et Aut $(M)$ le groupe de ses difféomorphismes holomorphes. Soit $G$ un groupe de Lie réel, simple et connexe. Soit $\Gamma$ un réseau de $G$. S'il existe un morphisme de $\Gamma$ dans $\operatorname{Aut}(M)$ dont l'image est infinie, le rang réel de $G$ est alors inférieur ou égal à la dimension complexe de $M$. 
Remarque 1.1. - L'inégalité sur le rang est plus forte que celle conjecturée par Zimmer car elle concerne la dimension complexe de $M$, mais cette amélioration ne doit pas être considérée comme une surprise puisque l'action est holomorphe.

Outre les travaux de Margulis et Zimmer, les points-clés de la démonstration du théorème $\mathrm{A}$ reposent sur

1. Les résultats de Salomon Bochner et Dean Montgomery concernant la structure du groupe des automorphismes des variétés complexes compactes, couplés à ceux de David I. Lieberman concernant les variétés kählériennes.

2. Un résultat récent de Tien-Cuong Dinh et Nessim Sibony concernant les actions holomorphes de groupes commutatifs sur les variétés kählériennes compactes.

C'est le résultat de Dinh et Sibony qui, associé à un théorème de Gopal Prasad et Madabusi S. Raghunathan, permet d'apporter la touche finale à la démonstration.

\section{Préliminaires}

Soit $M$ une variété complexe compacte. Par définition, les automorphismes de $M$ sont les difféomorphismes holomorphes de $M$; ils forment un groupe noté Aut $(M)$. Lorsque l'on munit ce groupe de la topologie de la convergence uniforme, le théorème de Bochner et Montgomery montre que $\operatorname{Aut}(M)$ est un groupe de Lie complexe dont l'algèbre de Lie s'identifie à celle des champs de vecteurs holomorphes de $M$ (voir [5]). Nous noterons $\operatorname{Aut}(M)^{0}$ la composante connexe de l'identité et $\operatorname{Aut}(M)^{\#}$ le quotient de $\operatorname{Aut}(M) \operatorname{par} \operatorname{Aut}(M)^{0}$.

\subsection{Exemples d'automorphismes}

Il se peut que $\operatorname{Aut}(M)^{\#}$ soit infini. C'est par exemple le cas pour une surface d'Enriques générique (voir [1]). Puisque toute la difficulté du théorème A provient justement de l'existence de tels groupes d'automorphismes, nous allons maintenant décrire quelques exemples explicites.

Soit $M$ une hypersurface lisse de $\left(\mathbb{P}^{1}(\mathbf{C})\right)^{k+1}$. Dans l'ouvert de Zariski $\mathbf{C}^{k+1}, M$ est définie par une équation polynomiale

$$
P\left(x_{1}, \ldots, x_{k+1}\right)=0 .
$$

Supposons que le degré du polynôme $P$ par rapport à chaque variable $x_{i}$ soit égal à deux; géométriquement, ceci signifie que chacune des projections

$$
\pi_{i}: M \rightarrow\left(\mathbb{P}^{1}(\mathbf{C})\right)^{k}
$$

consistant à oublier l'une des $k+1$ coordonnées est un revêtement ramifié de degré deux. D'après la formule d'adjonction, cette condition est équivalente à l'existence d'une $k$-forme holomorphe sur $M$ qui ne s'annule pas. Le théorème des sections hyperplanes de Lefschetz montre alors que $M$ est une variété de Calabi-Yau : la variété $M$ est kählérienne, compacte, simplement connexe et son fibré canonique est trivial. Une telle variété ne possède jamais de champs de vecteurs holomorphes et $\operatorname{Aut}(M)$ est donc un groupe discret.

Puisque chacun des revêtements ramifiés $\pi_{i}$ est de degré 2 , nous disposons de $k+1$ involutions holomorphes sur $M$ : chaque involution $\sigma_{i}$ permute les points des fibres de $\pi_{i}$. Il se trouve que le groupe engendré par ces involutions est isomorphe au produit libre de $k+1$ copies de $\mathbf{Z} / 2 \mathbf{Z}$. Autrement dit, il n'y a pas de relation non triviale entre ces involutions. Une démonstration de ce fait est proposée dans [7] pour le cas des surfaces et celle-ci s'étend en dimension quelconque. Le groupe d'automorphismes ainsi construit contient donc un groupe libre sur une infinité de générateurs. 
Lorsque $k$ est égal à 2 , la variété $M$ est une surface K3 avec une infinité d'automorphismes. Les produits symétriques $M^{[r]}$ de cette surface fournissent alors des exemples de variétés symplectiques de dimension arbitraire avec de nombreux automorphismes.

Il existe aussi des exemples surprenants sur certaines variétés rationnelles : si l'on éclate le plan projectif en les dix points doubles d'une sextique rationnelle générique, on obtient une surface projective dont le groupe d'automorphismes est isomorphe au produit libre de dix groupes abéliens libres de rang huit (voir [11]).

Pour des exemples d'actions de réseaux dans des groupes de Lie de rang supérieur, nous renvoyons le lecteur au paragraphe 3.1 .

\subsection{Cohomologie et composante connexe de l'identité}

Tout automorphisme $f$ d'une variété complexe compacte induit un isomorphisme $f^{*} \mathrm{du}$ groupe de cohomologie $H^{*}(M, \mathbf{Z})$. Cet isomorphisme préserve la structure de Hodge de $H^{*}(M, \mathbf{C})$. Lorsque $M$ est une variété kählérienne, on dispose d'un critère cohomologique pour déterminer les automorphismes isotopes à l'identité (voir [14], prop. 2.2).

THÉORÈME 2.1 (Lieberman). - Soient $M$ une variété complexe compacte kählérienne et $F$ un sous-groupe de $\operatorname{Aut}(M)$. Si F fixe une classe de Kähler, l'intersection de F avec Aut $(M)^{0}$ est un sous-groupe d'indice fini dans $F$.

En particulier, si l'action de $F$ sur la cohomologie de $M$ est triviale, l'intersection de $F$ avec $\operatorname{Aut}(M)^{0}$ est un sous-groupe d'indice fini dans $F$. Nous emploierons ce résultat au paragraphe 3.1. Ceci permettra de réduire la preuve du théorème $\mathrm{A}$ au cas des représentations à valeurs dans $\operatorname{Aut}(M)^{0}$.

\subsection{Variétés hyperkählériennes}

Une variété hyperkählérienne est une variété complexe compacte kählérienne qui est simplement connexe et qui possède une 2 -forme holomorphe symplectique. La dimension complexe de la variété est donc paire, égale à $2 d$, et il existe une 2 -forme holomorphe $\Omega$ telle que la $2 d$-forme $\wedge^{d} \Omega$ soit partout non nulle.

De nombreuses variétés de ce type possèdent un groupe d'automorphismes infini : c'est le cas, notamment, de certaines surfaces K3 et des produits symétriques associés (voir ci-dessus). Dans ce petit paragraphe, nous allons montrer directement le théorème $\mathrm{A}$ pour ce type de variétés. Ceci permettra d'illustrer et de préciser la démarche générale.

Proposition 2.2. - Soit $M$ une variété hyperkählérienne. Soit $G$ un groupe de Lie réel, simple et connexe dont le rang réel est supérieur ou égal à 2 . Soit $\Gamma$ un réseau de $G$. Tout morphisme de $\Gamma$ dans $\operatorname{Aut}(M)$ a une image finie.

Démonstration. - Toute variété hyperkählérienne $M$ est un produit de variétés hyperkählériennes irréductibles, ceci de manière unique à permutation près des facteurs (voir [2] et [3]). Si un groupe $\Gamma$ agit holomorphiquement sur $M$, un sous-groupe d'indice fini de $\Gamma$ préserve chaque facteur de ce produit. Nous pouvons donc supposer que $M$ est irréductible. En ce cas, la forme symplectique $\Omega$ est unique à un facteur multiplicatif près. En particulier, la forme $\Omega \wedge \bar{\Omega}$ est préservée par $\operatorname{Aut}(M)$.

Notons $q_{M}$ la restriction à $H^{1,1}(M, \mathbf{R})$ de la forme de Beauville (voir [2]); $q_{M}$ est donc la forme quadratique qui, à chaque forme $\alpha$ de type $(1,1)$, associe la quantité

$$
q_{M}(\alpha)=\int_{M} \alpha \wedge \alpha \wedge(\Omega \wedge \bar{\Omega})^{d-1},
$$


où $d$ est la moitié de la dimension complexe de $M$. Cette quantité ne dépend que de la classe de cohomologie de la forme $\alpha$ et détermine donc une forme quadratique sur $H^{1,1}(M, \mathbf{R})$. Il se trouve que la signature de cette forme est de type $(+,-, \ldots,-)$. Cette forme quadratique est invariante par l'action de $\operatorname{Aut}(M)$ car la décomposition de Hodge et la forme $\Omega \wedge \bar{\Omega}$ le sont.

Soit maintenant $G$ un groupe de Lie réel, simple, connexe et de rang réel supérieur ou égal à 2. Soit $\Gamma$ un réseau de $G$. Le groupe $\Gamma$ a donc la propriété (T) de Kazhdan et tout morphisme de $\Gamma$ dans $\mathrm{SO}(1, n)$ a donc une image relativement compacte (voir [8], cor. 23, p. 80).

Supposons que l'on dispose d'un morphisme de $\Gamma$ vers le groupe $\operatorname{Aut}(M)$. Le principe de Bochner montre que $\operatorname{Aut}(M)$ est discret; d'après le théorème 2.1, la proposition sera démontrée si nous montrons qu'un sous-groupe d'indice fini de $\Gamma$ fixe une classe de Kähler. Pour cela, il s'agit d'étudier l'action de $\Gamma$ sur $H^{1,1}(M, \mathbf{R})$. La remarque précédente et le calcul de la signature de $q_{M}$ montrent que l'image de $\Gamma$ dans $\operatorname{GL}\left(H^{1,1}(M, \mathbf{R})\right)$ est relativement compacte. Puisque les automorphismes de $M$ préservent la structure entière de l'homologie de $M$, l'action de $\Gamma$ sur $H^{1,1}(M, \mathbf{R})$ transite par un groupe fini. Il existe donc un sous-groupe d'indice fini dans $\Gamma$ qui fixe une classe de Kähler. Ceci termine la démonstration.

Remarque 2.1. - Cette proposition "montre" que les variétés hyperkählériennes se comportent comme les surfaces, ce que suggère déjà l'existence de la forme de Beauville.

\subsection{Un théorème de structure}

Nous aurons également besoin d'un théorème de structure pour $\operatorname{Aut}(M)^{0}$ relatif au morphisme d'Albanese. Avant de l'énoncer, rappelons quelques faits standards.

Soit $H^{0}\left(M, \Omega^{1}\right)$ le $\mathbf{C}$-espace vectoriel constitué des 1-formes holomorphes globales sur $M$. Lorsque $M$ est kählérienne et compacte, ce que nous supposons ici, toutes les formes holomorphes globales sont fermées. Soit $x$ un point de $M$. Pour chaque lacet $\gamma$ basé en $x$, l'intégration le long de $\gamma$ fournit une forme linéaire

$$
\omega \mapsto \int_{\gamma} \omega
$$

sur l'espace $H^{0}\left(M, \Omega^{1}\right)$ qui ne dépend que de la classe d'homologie de $\gamma$. La théorie de Hodge montre que $H_{1}(M, \mathbf{Z})$ détermine ainsi un réseau cocompact du dual de $H^{0}\left(M, \Omega^{1}\right)$. L'espace quotient, noté $\operatorname{Alb}(M)$, est la variété d'Albanese de $M$.

Si $y$ est un point de $M$, et si $\beta$ est un chemin joignant $x$ à $y$, l'intégration le long de $\beta$ ne dépend que de $y$ modulo des intégrales sur des lacets basés en $x$. Ce procédé définit donc une application holomorphe $\mathrm{a}_{M}: M \rightarrow \operatorname{Alb}(M)$, à savoir

$$
\mathrm{a}_{M}(y)=\int_{x}^{y} .
$$

Bien qu'elle dépende du choix d'un point base $x$, cette construction est équivariante sous l'action du groupe d'automorphismes de $M:$ il existe une représentation $\rho: \operatorname{Aut}(M) \rightarrow \operatorname{Aut}(\operatorname{Alb}(M))$ telle que

$$
\mathrm{a}_{M} \circ f=\rho(f) \circ \mathrm{a}_{M}
$$

pour tout automorphisme $f$.

L'énoncé dont nous ferons usage est le suivant (voir [14], thm. 3.12). 
THÉORÈME 2.3 (Lieberman).- Soient $M$ une variété complexe compacte kählérienne et $\mathrm{Alb}(M)$ la variété d'Albanese de $M$. Le noyau de la représentation

$$
\rho: \operatorname{Aut}(M)^{0} \rightarrow \operatorname{Aut}(\operatorname{Alb}(M))^{0}
$$

est un groupe algébrique complexe linéaire.

Remarque 2.2. - Le groupe $\operatorname{Aut}(\operatorname{Alb}(M))^{0}$ coïncide avec le groupe des translations de $\mathrm{Alb}(M)$. C'est donc un groupe abélien.

\subsection{Groupes commutatifs}

Dans [9], Dinh et Sibony démontrent le très joli théorème suivant.

THÉORÈme 2.4 (Dinh, Sibony). - Soient $M$ une variété complexe compacte kählérienne et $A$ un sous-groupe abélien de $\operatorname{Aut}(M)$. Si l'action de tout élément de $A \backslash\{i d\}$ sur la cohomologie de $M$ a un rayon spectral différent de 1 , alors $A$ est un groupe abélien libre dont le rang est strictement plus petit que la dimension complexe de $M$.

La démonstration utilise très astucieusement les inégalités de type Hodge-Riemann démontrées par Mickael Gromov à la suite des travaux de Bernard Teissier et Askold G. Khovanskii (voir [12]).

Remarque 2.3. - Le théorème que nous venons de citer n'est pas énoncé exactement sous cette forme dans [9]. Pour le déduire des résultats de Dinh et Sibony, il faut savoir qu'un automorphisme d'une variété kählérienne compacte a une entropie topologique strictement positive si, et seulement si son action sur la cohomologie de $M$ possède une direction propre dilatée (voir [9] et les références qui s'y trouvent).

\section{Démonstration du théorème $A$}

Dans cette partie, $M$ désigne une variété complexe compacte kählérienne, $G$ un groupe de Lie réel, simple et connexe et $\Gamma$ un réseau de $G$. Pour démontrer le théorème A, nous pouvons supposer, et nous le ferons, que le rang $r_{G}$ de $G$ est supérieur ou égal à 2 . Les deux références principales que nous utiliserons sont [17] et [19] car elles présentent les résultats dans un contexte proche du nôtre. Le lecteur peut bien sûr consulter [15], notamment les énoncés reproduits en page 4 .

\subsection{Action sur la cohomologie}

Proposition 3.1. - S'il existe un morphisme $\rho: \Gamma \rightarrow \operatorname{Aut}(M)$ tel que l'action induite sur la cohomologie de $M$ ait une image infinie, le rang réel $r_{G}$ est strictement plus petit que la dimension complexe de $M$.

Démonstration. - Par définition du rang, il existe un sous-groupe de Lie connexe $C$ de $G$ qui est abélien, de dimension $r_{G}$ et qui satisfait la propriété suivante : toute représentation linéaire de $C$ est constituée d'endomorphismes simultanément diagonalisables (prendre un tore multiplicatif maximal dans $G$ ).

D'après le théorème (2.8) de [16], il existe un élément $g$ de $G$ tel que $g \mathrm{Cg}^{-1}$ intersecte $\Gamma$ sur un groupe abélien libre de rang $r_{G}$, cocompact dans $g \mathrm{Cg}^{-1}$. Notons

$$
\rho^{*}: \Gamma \rightarrow \mathrm{GL}\left(\mathrm{H}^{*}(M, \mathbf{Z})\right)
$$


l'action de $\Gamma$ sur la cohomologie de $M$. Puisque $\rho^{*}(\Gamma)$ est infini et préserve la structure entière de la cohomologie, $\rho^{*}(\Gamma)$ n'est contenu dans aucun sous-groupe compact de $\mathrm{GL}\left(\mathrm{H}^{*}(M, \mathbf{C})\right)$. Le théorème de rigidité de Margulis affirme alors que $\rho^{*}$ s'étend en un morphisme de $G$ (voir [17], chap. 3, §6.6).

Le groupe $G$ étant simple, la restriction de ce morphisme à $g C g^{-1}$ réalise une bijection de $g C g^{-1}$ sur un sous-groupe diagonalisable de $\mathrm{GL}\left(\mathrm{H}^{*}(M, \mathbf{C})\right)$. Le groupe $\Gamma$ contient donc un sous-groupe abélien libre de rang $r_{G}$ dont les éléments distincts de l'identité agissent sur la cohomologie de $M$ avec un rayon spectral strictement plus grand que 1. Le théorème de Dinh et Sibony montre alors que $r_{G}$ est strictement inférieur à la dimension complexe de $M$.

Corollaire 3.2. - Supposons que $r_{G}$ est supérieur ou égal à la dimension complexe de M. Si $\rho: \Gamma \rightarrow \operatorname{Aut}(M)$ est un morphisme de groupes, il existe un sous-groupe d'indice fini de $\Gamma$ dont l'image par $\rho$ est contenue dans $\operatorname{Aut}(M)^{0}$.

Démonstration. - La proposition 3.1 montre que l'action de $\Gamma$ sur la cohomologie de $M$ a un noyau d'indice fini dans $\Gamma$. Le théorème 2.1 permet de conclure.

Exemple 3.1. - Soit $\Gamma$ le groupe $\operatorname{SL}(n, \mathbf{Z})$, vu comme réseau du groupe $\operatorname{SL}(n, \mathbf{R})$. Soit $\Lambda$ un réseau de $\mathbf{C}$ et $E$ la courbe elliptique $\mathbf{C} / \Lambda$. Le groupe $\Gamma$ agit linéairement sur $\mathbf{C}^{n}$ en préservant le réseau $\Lambda^{n}$. On obtient ainsi un morphisme injectif de $\Gamma$ vers le groupe des automorphismes de la variété abélienne $E^{n}$. L'action induite au niveau de la cohomologie est fidèle et l'on se situe dans le cas limite de la proposition 3.1 car le rang de $\mathrm{SL}(n, \mathbf{R})$ est égal à $n-1$.

Exemple 3.2. - Le groupe $\operatorname{PSL}(n+1, \mathbf{Z})$ s'injecte dans les automorphismes de l'espace projectif de dimension $n$. Ceci fournit un exemple pour le cas d'égalité dans le théorème A.

\subsection{Conclusion, première étape}

Vu le corollaire 3.2, nous pouvons désormais supposer que $\Gamma$ se plonge en un sous-groupe de $\operatorname{Aut}(M)^{0}$. Il s'agit de montrer que la dimension de $M$ est supérieure au rang réel de $G$. Ceci terminera la preuve du théorème $\mathrm{A}$.

Nous avons supposé que le rang réel de $G$ était supérieur ou égal à 2 , donc tout morphisme de $\Gamma$ dans un groupe résoluble a une image finie. En particulier, le groupe dérivé $[\Gamma, \Gamma]$ est d'indice fini dans $\Gamma$ (voir [17], chap. 3, thm. 7.1). Quitte à remplacer le réseau $\Gamma$ par son groupe dérivé, le théorème 2.3 permet de supposer que $\Gamma$ est "contenu" dans un sous-groupe $L \operatorname{de} \operatorname{Aut}(M)^{0}$ qui est un groupe algébrique complexe linéaire. Nous noterons

$$
\tau: \Gamma \rightarrow L \subset \mathrm{GL}(m, \mathbf{C})
$$

le plongement de $\Gamma$ associé.

Si l'image $\tau(\Gamma)$ n'est pas un sous-groupe relativement compact de $L$, nous pouvons étendre ce morphisme à $G$ de manière unique. Dans ce cas, $L$ est un groupe algébrique complexe de rang supérieur à celui de $G$ contenu dans $\operatorname{Aut}(M)^{0}$. Les inégalités

$$
r_{G} \leqslant r_{L} \leqslant \operatorname{dim}_{\mathbf{C}}(M)
$$

en découlent (voir [18], [19] §3, ou encore [6], §3).

\subsection{Conclusion, seconde étape}

Supposons désormais que $\tau(\Gamma)$ est un sous-groupe relativement compact de $L$. Nous allons construire une nouvelle représentation de $\Gamma$ dans $\operatorname{Aut}(M)^{0}$ dont l'image ne sera pas relativement 
compacte, ce qui terminera la démonstration via l'argument précédent. La méthode est classique : elle joue un rôle crucial dans l'alternative de Tits [4], le théorème 3.3 de [19] et le théorème 10.1 de [6].

Tout d'abord, nous pouvons supposer que $L$ est semi-simple. Soit, en effet, $R$ le radical résoluble de $L$ et $S$ le quotient de $L$ par $R$. Si la projection de $\tau(\Gamma)$ dans $S$ est finie, un sousgroupe d'indice fini de $\Gamma$ est contenu dans $R$. Puisque $R$ est résoluble, ceci est impossible. Quitte à changer $\Gamma$ en l'un de ses sous-groupes d'indice fini, la projection de $\tau(\Gamma)$ dans $S$ est donc un morphisme injectif. D'après le théorème de Levi-Malcev, la projection de $L$ sur $S$ admet une section. Le groupe $S$ apparait donc lui aussi comme un sous-groupe de $\operatorname{Aut}(M)^{0}$. Nous pouvons donc remplacer $L$ par $S$. Nous supposerons donc dorénavant que $L$ est un sous-groupe algébrique semi-simple de $\mathrm{GL}(m, \mathbf{C}), m \in \mathbf{N}$, et que $L$ est défini par des équations polynomiales à coefficients rationnels.

Le groupe $\Gamma$ étant un réseau dans le groupe connexe $G$, c'est un groupe de type fini (voir [17], chap. 1, §3.1). Fixons une partie génératrice finie $S$ de $\Gamma$ et notons $\mathcal{C}_{\tau}$ l'ensemble des coefficients des polynômes caractéristiques des matrices $\tau(\gamma)$, pour $\gamma$ dans $S$. Nous noterons $k(\tau)$ le corps $\mathbf{Q}\left(\mathcal{C}_{\tau}\right)$ engendré par ces coefficients.

\section{LEMME 3.3. - Le corps $k(\tau)$ est un corps de nombres.}

Remarque 3.1. - Soit $\sigma$ un isomorphisme du corps $k(\tau)$ sur un sous-corps de C. La représentation $\tau_{\sigma}$ obtenue en appliquant $\sigma$ à tous les coefficients des matrices $\tau(\gamma)$ est une nouvelle représentation de $\Gamma$ dans $L$, car $L$ est défini par des équations à coefficients dans $\mathbf{Q}$. Si $\lambda$ est une valeur propre de $\tau(\gamma)$, alors $\sigma(\lambda)$ est une valeur propre de $\tau_{\sigma}(\gamma)$.

Démonstration du lemme. - Soit $l$ le degré de transcendance de $k(\tau)$ sur Q. Il s'agit de montrer que $l$ est nul. Si ce n'est pas le cas, le théorème de l'élément primitif montre que $k(\tau)=\mathbf{Q}\left(x_{1}, \ldots, x_{l}\right)(y)$ où les $x_{i}$ sont des nombres complexes algébriquement indépendants et $y$ est algébrique sur $\mathbf{Q}\left(x_{1}, \ldots, x_{l}\right)$. Soit $P$ le polynôme minimal de $y$. Pour tout nombre réel strictement positif $\epsilon$, on peut choisir des nombres complexes algébriquement indépendants $x_{i}^{\prime}$ différents mais $\epsilon$-proches des $x_{i}$. Ceci détermine un isomorphisme $\sigma$ de $\mathbf{Q}\left(x_{1}, \ldots, x_{l}\right)$ sur $\mathbf{Q}\left(x_{1}^{\prime}, \ldots, x_{l}^{\prime}\right)$. Soit $P_{\sigma}$ le polynôme obtenu en appliquant $\sigma$ aux coefficients de $P$. Par continuité des racines, on peut choisir une racine $y^{\prime}$ de $P_{\sigma}$ proche de $y$. L'isomorphisme $\sigma$ s'étend donc en un plongement de $k(\tau)$ dans $\mathbf{C}$.

La remarque précédente permet alors de déformer la représentation $\tau$ en une représentation $\tau_{\sigma}$ qui est arbitrairement proche de $\tau$ car $\epsilon$ est arbitrairement petit. En outre, la représentation $\tau_{\sigma}$ n'est pas conjuguée à $\tau$ car $\sigma$ change les coefficients du polynôme caractéristique d'une des matrices $\tau(\gamma)$.

Le plongement de $\Gamma$ dans $L$ est localement rigide car, pour toute représentation $\nu$ de dimension finie en caractéristique nulle, le premier groupe de cohomologie de $\Gamma$ à valeurs dans $\nu$ est nul (voir [17], chap. 3, §7.1). On ne peut donc pas déformer localement la représentation $\tau$ autrement que par conjugaison.

Juxtaposées, ces deux propriétés montrent alors que $k(\tau)$ est un corps de nombres.

Le lemme de Selberg affirme que tout sous-groupe de type fini de $\mathrm{GL}(n, \mathbf{C})$ posséde un sousgroupe d'indice fini sans torsion (voir [17], §3.2). Étant donné que $\Gamma$ est un groupe de type fini infini, nous pouvons supposer que $\Gamma$ contient un élément $\gamma_{0}$ pour lequel $\tau\left(\gamma_{0}\right)$ est d'ordre infini. Comme l'image de $\Gamma$ est relativement compacte, la matrice $\tau\left(\gamma_{0}\right)$ est conjuguée à un élément de $\mathrm{U}(n, \mathbf{R})$; elle doit donc posséder une valeur propre $\lambda$ qui n'est pas une racine de l'unité.

Puisque $\lambda$ est solution d'une équation polynomiale à coefficients dans $k(\tau), \lambda$ est un nombre algébrique qui n'est pas une racine de l'unité. Le lemme de Kronecker assure alors l'existence 
d'un plongement $\alpha$ de $k(\tau)$ dans un corps local $k^{\prime}$ qui envoie $\lambda$ sur un nombre de norme plus grande que 1 (voir [13]).

En appliquant $\alpha$ aux coefficients des matrices $\tau(\gamma), \gamma \in \Gamma$, on construit une nouvelle représentation $\tau_{\alpha}: \Gamma \rightarrow L\left(k^{\prime}\right)$ dont l'image n'est plus relativement compacte. Ceci assure que $k^{\prime}$ est archimédien, c'est-à-dire que $k^{\prime}$ coïncide avec $\mathbf{R}$ ou $\mathbf{C}$ (voir [19], chap. 3, §3.2 ou [15], p. 4 , thm. $2^{\prime}$ ). Nous avons donc construit une nouvelle représentation de $\Gamma$ dans $L$ dont l'image n'est pas relativement compacte, ce qui termine la preuve du théorème $\mathrm{A}$.

\section{Compléments}

\subsection{Raffinements}

Le théorème $\mathrm{A}$ et sa démonstration ont plusieurs corollaires immédiats. On peut d'abord étendre le résultat à des variétés projectives sur d'autres corps que celui des nombres complexes.

ThÉORÈme B. - Soit $k$ un corps de caractéristique nulle. Soit $M$ une variété projective lisse sur $k$. Soit $G$ un groupe de Lie réel, simple et connexe. Soit $\Gamma$ un réseau de $G$. Si le rang réel de $G$ est strictement supérieur à la dimension de $M$, tout morphisme de $\Gamma$ dans le groupe des automorphismes de $M$ a une image finie.

Démonstration. - Soit $\rho: \Gamma \rightarrow \operatorname{Aut}(M)$ un morphisme. Le groupe $\Gamma$ est un groupe de type fini car c'est un réseau dans $G$. Nous noterons $\gamma_{1}, \ldots, \gamma_{l}$ un système de générateurs de $\Gamma$. L'ensemble des coefficients des polynômes qui définissent $M$ et les $\rho\left(\gamma_{i}\right)$ engendrent une extension de type finie de $\mathbf{Q}$. Nous pouvons donc supposer que le corps $k$ est une extension de type fini de $\mathbf{Q}$. Le corps $k$ peut donc être plongé en un sous-corps de $\mathbf{C}$ et le théorème se déduit ainsi du théorème $\mathrm{A}$.

Remarque 4.1. - Ce résultat me semble encore valable pour un corps de caractéristique positive, mais la démonstration nécessite d'étendre certains des théorèmes auxquels nous avons fait référence à un cadre plus général.

Il est également possible de généraliser le théorème A aux réseaux irréductibles de groupes semi-simples.

THÉORÈme C. - Soit $M$ une variété complexe compacte kählérienne de dimension complexe d. Soit $G$ un groupe de Lie réel, semi-simple et connexe. Soit $\Gamma$ un réseau irréductible de $G$.

S'il existe un morphisme $\rho: \Gamma \rightarrow \operatorname{Aut}(M)$ dont l'image est infinie, il existe un sous-groupe d'indice fini $\Gamma^{\prime}$ dans $\Gamma$ sur lequel $\rho$ est composé (i) du plongement de $\Gamma^{\prime}$ dans $G$, (ii) d'une surjection de $G$ sur un groupe de Lie $G^{\prime}$ de rang réel inférieur ou égal à d, (iii) d'une action de $G^{\prime}$ sur $M$ par difféomorphismes holomorphes.

Nous ne démontrerons pas ce résultat. Il se déduit facilement de la démonstration présentée dans la partie précédente.

\subsection{Questions}

Peu de variétés kählériennes compactes sont susceptibles d'admettre un groupe d'automorphismes dont le nombre de composantes connexes est infini. Il est donc envisageable, et il serait intéressant, de classer les variétés complexes compactes de dimension $d$ qui réalisent le cas d'égalité dans la proposition 3.1. On se donne donc un groupe de Lie réel, simple et connexe de rang réel $d-1$ et un réseau $\Gamma$ de $G$. Il s'agit alors de classer les couples $(M, \rho)$ où 
(i) $M$ est une variété complexe compacte kählérienne,

(ii) $\rho: \Gamma \rightarrow \operatorname{Aut}(M)$ est un morphisme de groupes,

(iii) l'action de $\rho(\Gamma)$ sur la cohomologie de $M$ ne transite pas par un groupe fini.

Il y a des exemples sur les tores et donc sur les variétés de Kummer qui s'en déduisent.

Si $M$ est projective et possède une telle action de réseau, alors sa dimension de Kodaira vaut $-\infty$ ou 0 , son premier groupe de cohomologie est un groupe de torsion ou son application d'Albanese est un isomorphisme, elle est rationnellement connexe (et donc simplement connexe) ou son fibré canonique est pseudo-effectif ... Toutes ces propriétés se déduisent facilement de résultats importants de géométrie algébrique complexe. Elles montrent que les deux cas principaux à étudier sont les variétés de Calabi-Yau et les variétés rationnellement connexes.

En conclusion, on peut dire qu'il serait intéressant de classer les variétés de Calabi-Yau de dimension 3 qui admettent une action holomorphe du groupe $\operatorname{SL}(3, \mathbf{Z})$. Le cas des variétés rationnellement connexes est également crucial, mais est sans doute le plus délicat : on ne sait toujours pas classer les surfaces rationnelles munies d'une action du groupe libre $\mathbf{Z} \star \mathbf{Z}$.

\section{Remerciements}

Un grand merci à Étienne Ghys pour m'avoir décrit, il y a quelques années, les difficultés et les stratégies liées à la conjecture de Zimmer, puis pour le soutien qu'il m'a apporté lors de la rédaction finale de ce texte.

Mon intérêt pour la conjecture de Zimmer dans un contexte holomorphe fut ravivé par les discussions que j' ai eues avec Francois Labourie lors du colloque pour les soixante ans d'Alberto Verjovsky. Merci à F. Labourie et aux organisateurs de ce colloque.

Merci au rapporteur et à Bruno Klingler pour leur relecture attentive de ce texte.

\section{RÉFÉRENCES}

[1] Barth W., Peters C., Automorphisms of Enriques surfaces, Invent. Math. 73 (3) (1983) $383-411$.

[2] Beauville A., Variétés kählériennes dont la première classe de Chern est nulle, J. Differential Geom. 18 (4) (1984) 755-782, 1983.

[3] BeAuville A., Riemannian holonomy and algebric geometry, preprint, math.AG/9902110, 1999, pp. 1-27.

[4] BENOIST Y., Sous-groupes discrets des groupes de Lie, in: European Summer School in Group Theory, 1997, pp. 1-72.

[5] Bochner S., Montgomery D., Locally compact groups of differentiable transformations, Ann. of Math. (2) 47 (1946) 639-653.

[6] CAIRns G., GHYs É., The local linearization problem for smooth SL(n)-actions, Enseign. Math. (2) 43 (1-2) (1997) 133-171.

[7] Cantat S., Dynamique des automorphismes des surfaces K3, Acta Math. 187 (1) (2001) 1-57.

[8] DE la Harpe P., VAletTe A., La propriété $(T)$ de Kazhdan pour les groupes localement compacts avec un appendice de Marc Burger, Astérisque 175 (1989) 158. With an appendix by M. Burger.

[9] Dinh T.-C., SibONy N., Groupes commutatifs d'automorphismes d'une variété kählérienne compacte, Duke Math. J. 123 (2004) 311-328.

[10] GHys É., Actions de réseaux sur le cercle, Invent. Math. 137 (1) (1999) 199-231.

[11] Gizatullin M.H., Rational G-surfaces, Izv. Akad. Nauk SSSR Ser. Mat. 44 (1) (1980) 110-144, 239.

[12] Gromov M., Convex sets and Kähler manifolds, in: Advances in Differential Geometry and Topology, World Sci. Publishing, Teaneck, NJ, 1990, pp. 1-38.

[13] Hindry M., Silverman J.H., Diophantine Geometry, Graduate Texts in Mathematics, vol. 201, Springer-Verlag, New York, 2000. An introduction. 
[14] Lieberman D.I., Compactness of the Chow scheme: applications to automorphisms and deformations of Kähler manifolds, in: Fonctions de plusieurs variables complexes, III (Sém. François Norguet, 1975-1977), Springer, Berlin, 1978, pp. 140-186.

[15] Margulis G.A., Discrete Subgroups of Semisimple Lie Groups, in: Ergebnisse der Mathematik und ihrer Grenzgebiete (3), vol. 17 (Results in Mathematics and Related Areas (3)), Springer-Verlag, Berlin, 1991.

[16] Prasad G., Raghunathan M.S., Cartan subgroups and lattices in semi-simple groups, Ann. of Math. 296 (1972) 296-317.

[17] Vinberg E.B., Gorbatsevich V.V., ShVartsman O.V., Discrete subgroups of Lie groups, in: Lie Groups and Lie Algebras, II, in: Encyclopaedia Math. Sci., vol. 21, Springer, Berlin, 2000, pp. 1-123, 217-223. [MR 90c:22036].

[18] Zimmer R.J., Actions of semisimple groups and discrete subgroups, in: Proceedings of the International Congress of Mathematicians, vols. 1, 2, (Berkeley, CA, 1986), Amer. Math. Soc., Providence, RI, 1987, pp. 1247-1258.

[19] ZIMMER R.J., Lattices in semisimple groups and invariant geometric structures on compact manifolds, in: Discrete Groups in Geometry and Analysis (New Haven, CT, 1984), in: Progr. Math., vol. 67, Birkhäuser Boston, Boston, MA, 1987, pp. 152-210.

\section{Serge CANTAT}

IRMAR,

Université de Rennes,

Campus de Beaulieu,

35042 Rennes Cedex, France

E-mail : cantat@maths.univ-rennes1.fr 